\title{
Resíduo de fruta cítrica como precursor de Carbon dots: um estudo de reprodutibilidade
}

Citrus fruit waste as precursor of Carbon dots: a study of reproducibility

A. B. Dos Santos; J. C. Nascimento; I. M. Oliveira; T. V. Dos Santos; C. D. A. E. S. Barbosa*

Instituto de Química e Biotecnologia/Grupo de Reatividade Química e Catálise (GCaR), Universidade Federal de Alagoas, 57072-900, Maceió- AL, Brasil

*cintya.barbosa@iqb.ufal.br

(Recebido em 13 de outubro de 2019; aceito em 13 de fevereiro de 2020)

\begin{abstract}
Nos últimos anos, a busca por precursores verdes para a síntese de nanopartículas de carbono (carbon dots), tem despertado interesse da comunidade científica, devido dentre outros aspectos, o alinhamento com a química verde, abundância de resíduos carbonáceos e ampla faixa de aplicações desses nanomateriais. Nesse sentido, uma estratégia simples, verde e de baixo custo, foi desenvolvida para a síntese reprodutível de carbon dots (C-dots) usando extrato do bagaço de limão, proveniente de diferentes cidades de Alagoas, Brasil, a saber Rio Largo, Coruripe e Maceió. As dispersões aquosas das nanopartículas, exibiram emissão no verde, tamanhos médios abaixo de $10 \mathrm{~nm}$ e índice de polidispersividade entre $0-1$, indicando uma distribuição monodispersa das nanopartículas. As análises de FTIR e absorção UV-Vis evidenciaram a presença de estiramentos vibracionais de grupos funcionais oxigenados e bandas de absorção atribuídas as transições $\pi$ $\pi^{*}(230 \mathrm{~nm})$ e $\mathrm{n}-\pi^{*}$ em $(286 \mathrm{~nm})$, respectivamente. Os espectros de fotoluminescência, mostraram que a emissão dos C-dots é dependente do comprimento de onda de excitação, exibindo uma intensidade máxima em $445 \mathrm{~nm}$, quando excitados em $350 \mathrm{~nm}$. Assim, foi observada uma boa correlação das propriedades ópticas e estruturais dos C-dots, indicando a reprodutibilidade dessas nanopartículas. Além disso, os C-Dots exibiram potencial aplicação como sensor de cromo (VI), demonstrando que o uso do extrato do bagaço de limão é uma forma eficiente de produzir C-dots de baixo custo via química verde.

Palavras-chave: síntese verde, carbon dots, reprodutibilidade.
\end{abstract}

In recent years, the search for green precursors for the synthesis of carbon nanoparticles (Carbon Dots) has attracted the attention of the scientific community due, among other factors, to the alignment with green chemistry, abundance of carbonaceous residues and a broad range of applications of these nanomaterials. In this sense, a simple, green and low-cost strategy was developed for the reproducible synthesis of C-Dots using lemon bagasse extract from different cities of Alagoas, Brazil, including Rio Largo, Coruripe e Maceió. The aqueous dispersions of the nanoparticles exhibited green emission and average sizes below $10 \mathrm{~nm}$ and polydispersivity index between $0-1$, indicating a monodisperse distribution of the nanoparticles. The FTIR and UV-Vis analyses revealed the presence of vibrational stretching of oxygenated functional groups and absorption bands attributed to transitions $\pi-\pi^{*}(230 \mathrm{~nm})$ and $\mathrm{n}-\pi^{*}(286 \mathrm{~nm})$, respectively. The photoluminescence spectra revealed that the emission of C-Dots is dependent on the excitation wavelength, with a maximum intensity at $445 \mathrm{~nm}$ when excited at $350 \mathrm{~nm}$. Thus, a great correlation of optical and structural properties of C-dots was evidenced, indicating the reproducibility of these nanoparticles. In addition, C-Dots exhibited potential application as a chromium (VI) sensor, showing that the use of lemon extract is an efficient way to produce low-cost C-dots via green chemistry.

Keywords: green synthesis, carbon dots, reproducibility.

\section{INTRODUÇÃO}

Desde a sua descoberta acidentalmente em 2004, durante uma purificação rotineira de nanotubos de carbono [1,2], os pontos quânticos de carbono, ou simplesmente carbon dots (C-Dots), vêm atraindo atenção de pesquisadores do mundo inteiro, por ser um novo tipo de nanomaterial de carbono, que exibe luminescência, biocompatibilidade, tamanhos inferiores a $10 \mathrm{~nm}$, solubilidade em água e que podem ser obtidos por rotas sintéticas de baixo custo [3, 4]. Em virtude das suas propriedades ópticas únicas, os C-Dots têm sido aplicados em diferentes áreas, a exemplo das biológicas, ambientais e ópticas-eletrônicas $[3,5,6]$. O mecanismo de luminescência dos C-Dots ainda não é totalmente elucidado, uma vez que são produzidos por diversos precursores e diferentes 
rotas sintéticas, além de pós-tratamentos, sendo assim, apresentam desempenhos ópticos diversos [4]. Conquanto, os mecanismos de luminescência mais aceitos atualmente, são os de estado de superfície [4, 7], efeito de confinamento quântico [4, 8] e fluorescência molecular [9, 10].

As sínteses dos C-Dots podem ser realizadas através de dois métodos comuns para nanopartículas, a saber o top-down e bottom up [4]. No método top-down (de cima pra baixo), são utilizados geralmente materiais como grafite, carbono orgânico, óxido de grafeno e nanotubos de carbono, os quais são reduzidos a carbon dots, via processos de oxidação eletroquímica, ablação a laser e ultrassom [4]. Por outro lado, o método bottom-up (de baixo pra cima), visa a formação de C-Dots a partir de moléculas, a exemplo do ácido cítrico, aminoácidos, carboidratos, entre outros, através de processos via hidrotermal, solvotermal, microondas, e etc $[4,11]$. Desde que Hsu et al.(2012) [12] publicaram pela primeira vez uma síntese de carbon dots derivados das borras de café, essas nanopartículas vêm sendo sintetizadas através de materiais carbonáceos residuais, os quais são transformados em fontes verdes de carbono, tornando-se uma alternativa simples, sustentável, rápida e econômica para a ciência na produção de C-Dots. Segundo Sharma et al. (2017) [11] esses precursores podem ser derivados de animais, humanos, alimentos e materiais residuais. Em vista disso, devido o limão ser uma das frutas mais consumidas do mundo, tornou-se um dos resíduos mais explorados na síntese verde de C-Dots, onde são utilizadas suas cascas, sucos e extratos $[13,14,15,16]$. Esses resíduos, têm em sua composição ácido cítrico, ácido ascórbico, vitaminas do complexo $\mathrm{B}$, flavonoides, sais minerais e pequenas concentrações de compostos nitrogenados e substâncias pécticas $[17,18]$.

Na literatura, são reportadas diversas sínteses usando limão como precursor de C-Dots. Ding et al. (2017) [14] relataram uma síntese solvotermal com suco de limão e etanol, que após uma purificação em coluna cromatográfica com acetato de etila e metanol, obteve-se um carbon dot com emissão no vermelho, onde foram aplicados em ensaios de bioimagem. Basavaiah et al. (2018) [15] sintetizaram carbon dots do suco de limão com etanol, obtendo um C-Dots com luminescência no azul, o qual foi aplicado em imageamento celular. Comumente, na literatura, utiliza-se o suco de limão como precursor, sendo menos frequente o uso das suas cascas [13]. Ademais, os trabalhos que envolvem o uso dessas, utilizam solventes tóxicos e/ou rotas sintéticas menos brandas, a exemplo de Tyagi et al. (2016) [16], os quais sintetizaram C-Dots a partir de resíduos das cascas de limão, por meio de um processo hidrotermal, onde foram imobilizados com o $\mathrm{TiO}_{2}$, para o desenvolvimento de uma sonda fluorescente, para deteç̧ão de $\mathrm{Cr}$ (VI). Adicionalmente, ensaios de reprodutibilidade são de grande relevância quando se realiza sínteses de C-Dots utilizando precursores verdes, uma vez que a composição desses, podem sofrer variações devido ao clima, localidade, diferentes espécies, rotas sintéticas, dentre outros $[4,11]$. Como Zhang et al. (2015) [19], a fim de sintetizar carbon dots em larga escala e reprodutíveis, relataram uma síntese verde, utilizando três tipos diferentes de poléns de abelha, onde obtiveram C-dots com emissão no azul e propriedades ópticas e estruturais similares, sugerindo a reprodutibilidade das nanopartículas. Meiling et al. (2016) [20] também testaram a reprodutibilidade de carbon dots usando amido como precursor de carbono, onde obtiveram C-Dots reprodutíveis em seus aspectos ópticos.

Por fim, neste trabalho, foram realizadas três sínteses de C-Dots através de uma via hidrotermal, usando o resíduo carbonáceo resultante do extrato do bagaço de limão do tipo taiti (Citrus latifolia), provenientes de diferentes cidades de Alagoas, a fim de avaliar preliminarmente a reprodutibilidade dessas nanopartículas. Os C-Dots produzidos foram caracterizados em seus aspectos estruturais e fotofísicos e demonstraram potenciais em aplicações como sensores de cromo (VI).

\section{MATERIAL E MÉTODOS}

\subsection{Síntese dos carbon dots}

Limões do tipo taiti (Citrus latifolia), foram cortados em 4 partes, e em seguida, espremidos com o auxílio de um espremedor de limão doméstico para obtenção do suco e do bagaço (contendo as cascas). O bagaço foi adicionado a um béquer com água deionizada $(100 \mathrm{~mL})$, o qual foi posto em uma chapa de aquecimento durante $60 \mathrm{~min}$ a $100^{\circ} \mathrm{C}$, para obtenção do extrato (Figura 1). Posteriormente, realizou-se uma filtração simples para separar o bagaço do extrato aquoso. Deste último, transferiu-se $14 \mathrm{MI}$ para um reator de teflon, revestido de aço inox. Prontamente, o reator 
foi colocado na mufla, onde permaneceu por $6 \mathrm{~h}$ a $180^{\circ} \mathrm{C}$. Após esse tempo, o C-Dots formado, foram centrifugados a $10000 \mathrm{RPM}$ durante 10 minutos e filtrados em membranas de $0,20 \mu \mathrm{m}$. Seguidamente, as dispersões aquosas dos C-Dots sem diluição adicional $(68 \mathrm{mg} / \mathrm{mL})$, foram transferidas para tubos falcon e armazenadas em geladeira $\left(\approx 6^{\circ} \mathrm{C}\right)$, até o instante de análise. Os procedimentos descritos acima, foram realizados para limões provenientes de diferentes cidades de Alagoas, a fim de realizar os testes de reprodutibilidade entre as cidades. Para tanto, os carbon dots foram designados pelas siglas C-Dots_AMx para cidade de Maceió, C-Dots_ACx para cidade de Coruripe e C-Dots_ARx para cidade de Rio Largo, onde x assume valores de 1, 2 e 3, os quais estão relacionados as sínteses em triplicata e em dias diferentes para cada cidade. Os carbon dots sintetizados são hidrofílicos, e, portanto, altamente solúveis e estáveis em água, apresentando-se como uma solução de coloração amarela. As dispersões aquosas dos carbon dots irradiados à luz UV, apresentaram luminescência no verde (Figura 1).

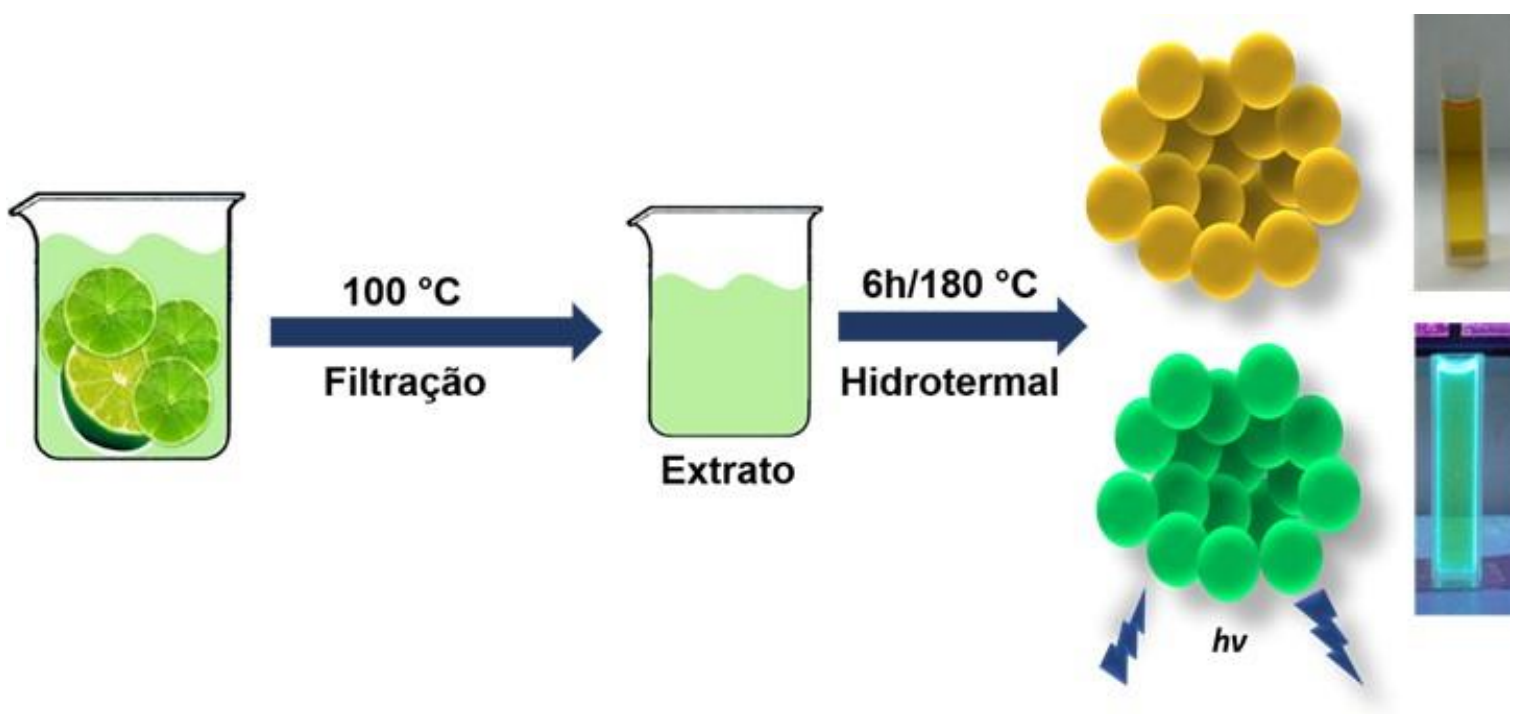

Figura 1: Esquema ilustrativo da sintese dos carbon dots (imagens das nanopartículas em solução e sob lâmpada UV).

\subsection{Caracterização dos carbon dots}

A composição química dos carbon dots sintetizados foi analisada por meio da espectroscopia de infravermelho transformada de Fourier (FTIR) usando o Termo Scientific modelo Nicolet e IR1200. Os espectros de absorbância foram adquiridos utilizando-se de um espectrofotômetro UVVIS modelo UV-3600 Plus, numa faixa de absorção de 200 a $1000 \mathrm{~nm}$, utilizando cubetas de quartzo com caminho óptico de $1,0 \mathrm{~cm}$. As medidas de distribuição das partículas dispersas foram feitas por meio da técnica de espalhamento dinâmico de luz (DLS), através do equipamento Microtrac Zetatrac 150 Particle Counter, séries S3000/S3500 à $25^{\circ} \mathrm{C}$, que por sua vez, baseia-se no movimento Browniano, onde seu tamanho pode ser obtido através de uma relação entre o coeficiente de difusão e o raio hidrodinâmico da partícula. O índice de polidispersidade (PDI) que fornece informações com relação ao grau de homogeneidade de uma determinada amostra, foi determinado por meio das medidas de DLS, no ângulo de $180{ }^{\circ} \mathrm{C}$, sendo calculado com base na Equação 1 [21].

$$
P D I=\frac{\sigma^{2}}{D m^{2}}
$$

Onde $\sigma$ é o desvio padrão da distribuição de tamanho (em nm) e Dm é o diâmetro médio (em $\mathrm{nm}$ ) da partícula. As análises dos espectros de emissão foram realizadas com o auxílio de um Espectrofluorímetro Fluorolog 3-22 HORIBA com comprimento de onda de excitação variando de 290 a $410 \mathrm{~nm}$. 


\section{RESULTADOS E DISCUSSÃO}

É apresentada uma síntese hidrotermal, simples e verde de C-Dots utilizando o extrato do bagaço de limão taiti em meio aquoso, como precursor carbonáceo. Os resultados dos histogramas dos CDots_AR1-3, C-Dots_AM1-3 e C-Dots_AC1-3 adquiridos pelo DLS, exibiram uma distribuição de tamanho médio abaixo de $10 \mathrm{~nm}$, apresentando valores de 4,64 \pm 0,344, 4,70 \pm 0,050 e 5,41 \pm $1.386 \mathrm{~nm}$ para o C-Dots_AM1-3, C-Dots_AC1-3 e C-Dots_AR1-3, referentes as cidades de Maceió, Coruripe e Rio Largo, respectivamente (Figuras 2 A-I). Os índices de polidispersividade exibiram valores entre 0 e 1 , indicando uma distribuição monodispersa das nanopartículas de carbono (Tabela 1).
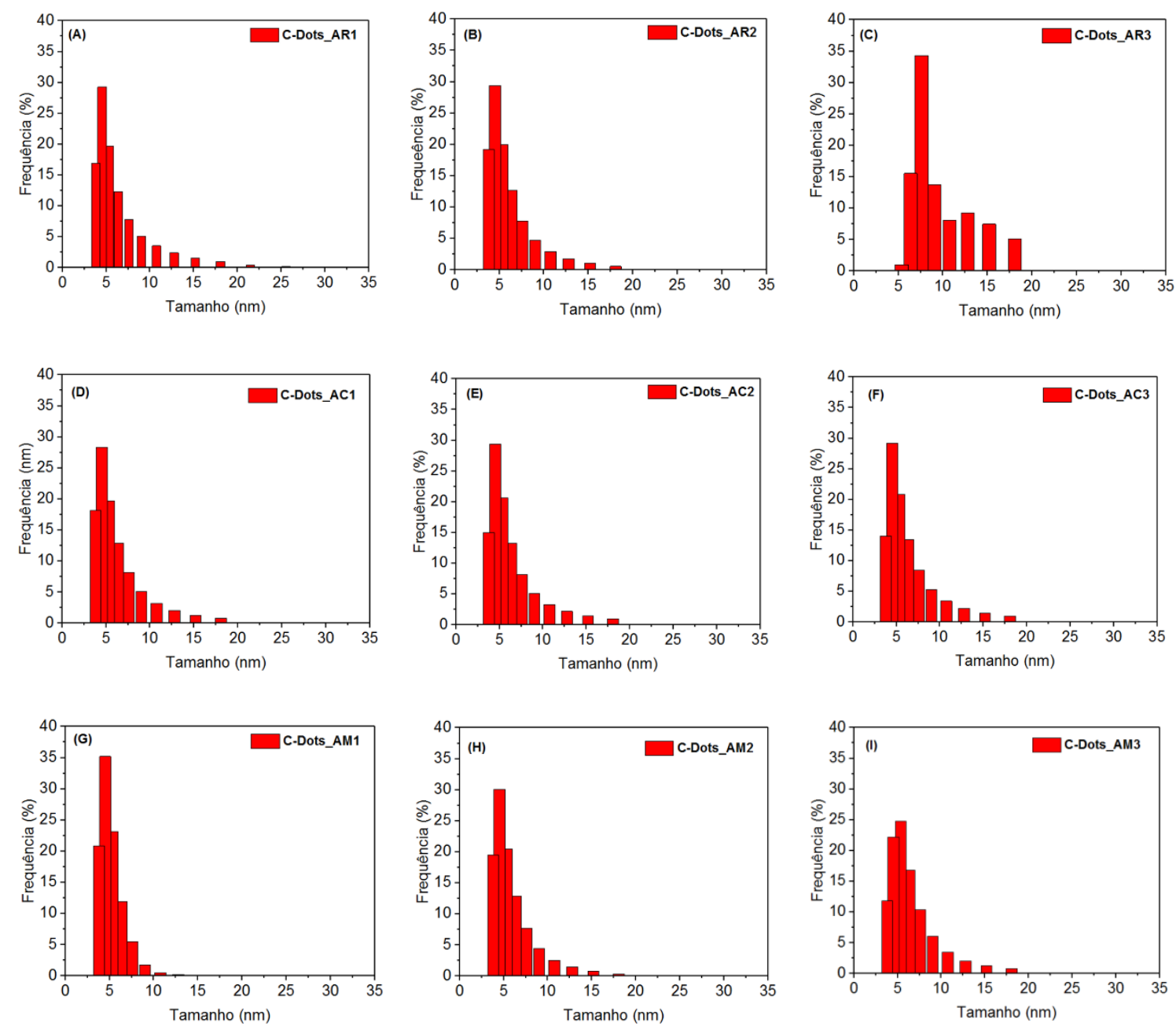

Figura 2: Histogramas da distribuição de tamanho médio dos (A) C-Dots_AR1, (B) C-Dots_AR2, (C) CDots_AR3, (D) C-Dots_AC1, (E) C-Dots_AC2 (F) C-Dots_AC3, (G) C-Dots_AM1, (H) C-Dots_AM2 e (I) $C$-Dots_AM3.

Tabela 1: Medidas de distribuição média das nanopartículas dispersas e índice de polidispersividade

\begin{tabular}{ccc}
\hline Amostras & Diâmetro médio (nm) & PDI \\
\hline C-Dots_AM & $4,64 \pm 0,344$ & 0,0050 \\
C-Dots_AC & $4,70 \pm 0,050$ & 0,0001 \\
C-Dots_AR & $5,41 \pm 1.386$ & 0,0656 \\
\hline
\end{tabular}

Os C-Dots obtidos foram caracterizados por espectrofotometria UV-Vis (Figuras 3 A-C). Os espectros dos C-Dots revelaram duas bandas de absorção características, centradas em 230 e 286 nm. A primeira banda de absorção, localizada na faixa de $190-250 \mathrm{~nm}$ é atribuída à transição eletrônica $\pi-\pi *$ das ligações $C=C$ (domínios $\mathrm{sp}^{2}$ ). A segunda faixa de absorção $(250-400 \mathrm{~nm}$ ), 
corresponde à transição eletrônica $\mathrm{n}-\pi *$ das ligações $\mathrm{C}=\mathrm{O}[13,14,15]$. Todos os carbon dots sintetizados tiveram o mesmo perfil das bandas de absorção.
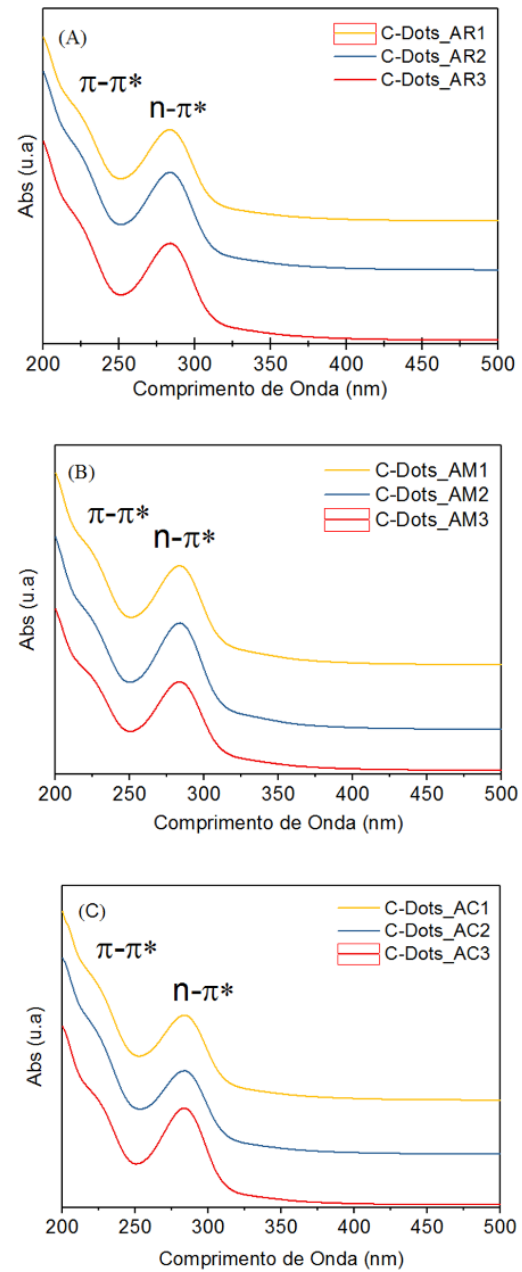

Figura 3: Espectros de UV-Vis dos C-Dots_AR1-3, C-Dots_AM1-3 e C-Dots_AC1-3.

Para descrever os grupos funcionais presentes na superfície dos C-Dots, a espectroscopia de infravermelho com transformada de Fourier (FTIR) foi realizada e apresentaram perfis similares (Figura 4). Os C-Dots_A1, C- Dots_A2 e C-Dots_A3 exibiram bandas largas e intensas em 3750 $\mathrm{cm}^{-1}$ e $3180 \mathrm{~cm}^{-1}$ que podem ser atribuídas as vibrações de estiramento das ligações $\mathrm{O}-\mathrm{H}$ carboxílicas e $\mathrm{N}-\mathrm{H}[4,13,14,15]$. Em $1712 \mathrm{~cm}^{-1}$ observa-se o estiramento $\mathrm{C}=\mathrm{O}$, confirmando a presença de ácido carboxílico nos carbon dots sintetizados, indicando que grupos funcionais oxigenados, estão presentes na superfície dos C-Dots [13, 14]. Bandas de estiramentos C-H aparecem em $2940 \mathrm{~cm}^{-1}$, já em $1391 \mathrm{~cm}^{-1}$ observa-se as vibrações de estiramentos $\mathrm{C}=\mathrm{C}$, indicando a formação de poliaromáticos $[4,13]$. Estiramentos vibracionais das ligações $\mathrm{C}-\mathrm{N}$ aparecem em $1180 \mathrm{~cm}^{-1}$, os quais podem ser atribuídos as estruturas das vitaminas B1 e B2 presentes no extrato. A alta solubilidade em água dos C-Dots é resultado desses diferentes grupos funcionais na superfície $[4,11]$. 

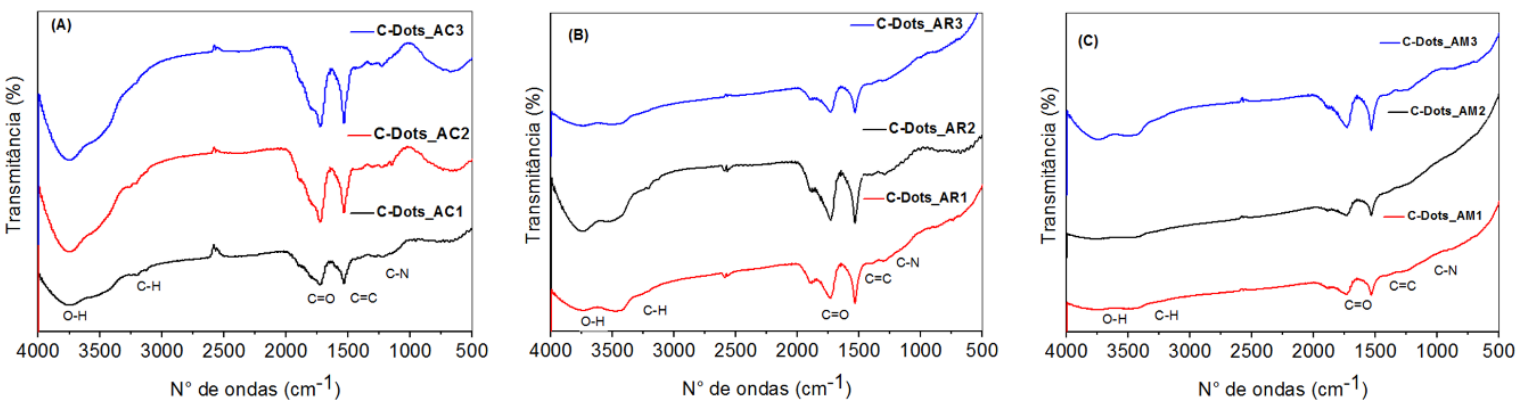

Figura 4: Espectros de infravermelho com transformada de Fourier (FTIR) para os C-Dots_AC1-3, CDots_ARI-3 e C-Dots_AM1-3.

Os espectros de emissão de fluorescência das nanopartículas foram registrados em diferentes comprimentos de onda de excitação, os quais variaram na faixa de 290 - $410 \mathrm{~nm}$ (Figuras 5 A - I). Uma das principais características luminescentes dos carbon dots, encontradas em vários trabalhos da literatura, foi observada nesse estudo, a saber, emissão dependente do comprimento de onda de excitação $[13,14,20]$. A emissão dos C-Dots apresentou um desvio para o vermelho à medida que o comprimento de onda de excitação aumentava de 290 para $410 \mathrm{~nm}[4,13]$.
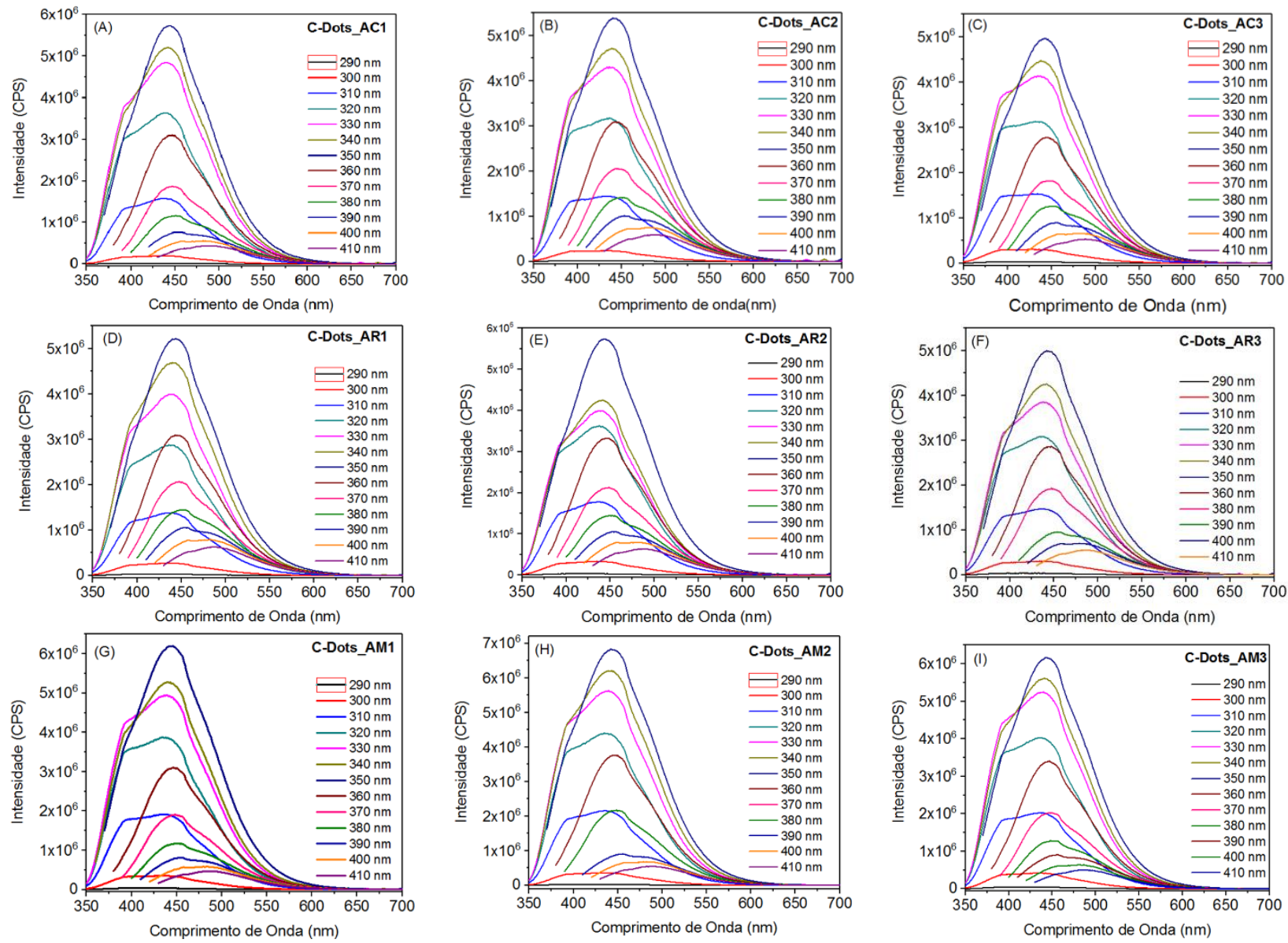

Figura 5: Espectros emissão dos para os (A) C-Dots_AC1, (B) C-Dots_AC2, (C) C-Dots_AC3, (D) CDots_AR1, (E) C-Dots_AR2 (F) C-Dots_AR3, (G) C-Dots_AM1, (H) C-Dots_AM2 e (I) C-Dots_AM3 excitados numa faixa de 290-410 $\mathrm{nm}$.

Ao analisar os espectros de emissão dos carbon dots de cada cidade (Maceió, Coruripe e Rio Largo), observou-se que os nanomateriais exibiram o mesmo perfil espectral com emissão máxima centrada em $445 \mathrm{~nm}$ (quando excitados em $350 \mathrm{~nm}$ ). Esse perfil similar das bandas de emissão dos 
diferentes carbon dots, sugerem uma reprodutibilidade quanto as propriedades ópticas dessas nanopartículas provenientes de diferentes cidades de Alagoas.

Embora a literatura reporte carbon dots que exibem emissão independente do comprimento de onda de excitação $\lambda_{\text {exc }}$ (seguindo a regra de Kasha), o mecanismo de emissão dependente do $\lambda_{\text {exc }}$ é ainda o fenômeno mais observado e melhor elucidado para carbon dots. [4] Este comportamento tem sido reportado por alguns pesquisadores como uma função dos estados de superfície, os quais são associados a grupos funcionais (em muitos casos estados moleculares) como $\mathrm{C}=\mathrm{O}, \mathrm{N}-\mathrm{H}, \mathrm{C}=\mathrm{N}$ e etc. [14] Esses grupos de superfície podem introduzir diferentes níveis de energia nos carbon dots e promover a fluorescência com diferentes comprimentos de onda de emissão $\left(\lambda_{\mathrm{em}}\right)$. As propriedades luminescentes dos carbon dots relativas aos defeitos de superfície, confinamento quântico, grau de oxidação e/ou mudança composicional são os mecanismos mais comuns associados a emissão de carbon dots derivados de limão [13-17, 22]. Ding et al. (2017) [14] relataram uma síntese de carbon dots com emissão no vermelho utilizando suco de limão na presença de diferentes quantidades de etanol. A emissão no vermelho foi atribuída ao aumento de nitrogênio na estrutura das nanopartículas, o qual foi induzido pela presença de etanol. Adicionalmente, o efeito de confinamento quântico foi descartado, uma vez que os tamanhos dos carbon dots permaneciam praticamente inalterados frente as modificações composicionais. Entretanto, no trabalho reportado por Hoan et al. (2019) [22], a emissão com deslocamento para o vermelho e dependente do $\lambda_{\text {exc }}$ foi atribuída ao efeito de confinamento quântico. Neste caso, as nanopartículas aumentavam de tamanho mediante o aumento da temperatura de síntese, e exibiam emissão em comprimento de onda mais longos.

Estudos preliminares de aplicação das nanopartículas de carbono como sensores fluorescentes de íons potencialmente tóxicos foram investigados visando avaliar se existe um perfil similar de resposta analítica para os C-Dots provenientes de diferentes cidades (Figuras $6 \mathrm{~A}-\mathrm{C}$ ).
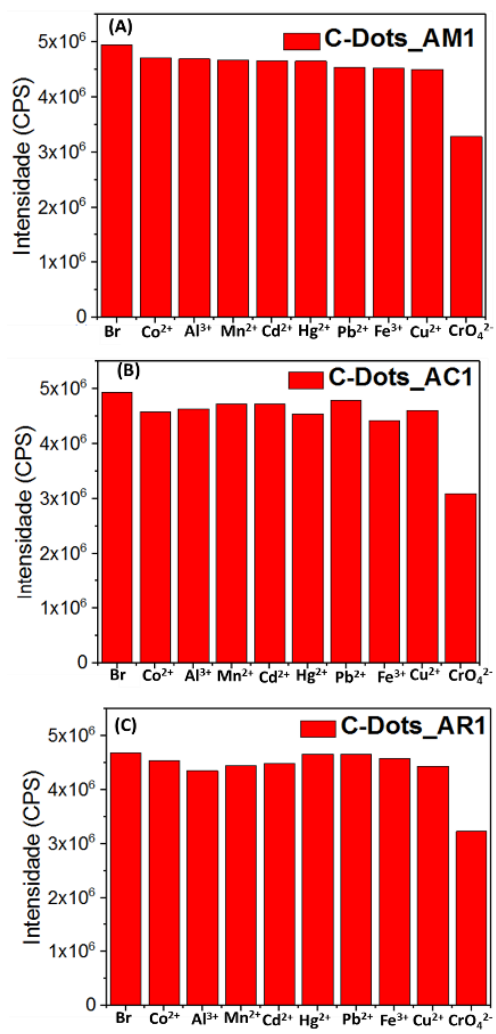

Figura 6: Intensidade de fluorescência para os (A) C-Dots_AMI, (B) C-Dots_ACle (C)C-Dots_ARI com diferentes ions metálicos $300 \mu M\left(\lambda_{\text {exc }}=350 \mathrm{~nm}\right)$.

Para os ensaios de sensoriamento, preparou-se $3 \mathrm{~mL}$ de uma solução aquosa contendo $100 \mu \mathrm{L}$ dos C-Dots (C-Dots_AC1, C-Dots_AM1 e C-Dots_AR1) na concentração de $6,8 \mathrm{mg} / \mathrm{mL}$ com 90 $\mu \mathrm{L}$ de diferentes íons metálicos $\left(\mathrm{Al}^{3+}, \mathrm{Co}^{2+}, \mathrm{Cd}^{2+}, \mathrm{Cr}^{6+}, \mathrm{Mn}^{2+}, \mathrm{Ni}^{2+}, \mathrm{Pb}^{2+}, \mathrm{Hg}^{2+}, \mathrm{Cu}^{2+}\right.$ e $\left.\mathrm{Fe}^{3+}\right)$ 
preparados em condições idênticas $\left(\mathrm{pH}=5,5,24^{\circ} \mathrm{C}\right.$ e mesmo contra-íons) com concentração final de $300 \mu \mathrm{M}$. As soluções foram excitadas em $350 \mathrm{~nm}$, e a intensidade de emissão centrada em 445 $\mathrm{nm}$ foi monitorada quanto a presença dos íons metálicos em relação a amostra de referência (Branco). Os resultados indicaram que para todos os carbon dots a resposta analítica frente a diferentes íons metálicos foi a mesma, ou seja, os C-Dots provenientes de diferentes cidades exibiram uma supressão de fluorescência mais significativa para o cromo (VI), o qual tem sua concentração controlada em diversos compartimentos aquáticos (incluindo água potável) por ser potencialmente tóxico e cancerígeno [16].

Nesse sentido, para o nosso conhecimento, esse tipo de ensaio que relaciona reprodutibilidade com aplicações dos C-Dots, em especifico na detecção de íons metálicos, ainda não foi reportada na literatura. Desse modo, tal estudo vem a contribuir em novos trabalhos voltados para área de sensores analíticos utilizando C-Dots provenientes de precursores verdes, no sentido em que permite uma maior garantia e confiabilidade dos dados, bem como uma melhor projeção para aplicação futura em sistemas reais.

\section{CONCLUSÃO}

Foi desenvolvida com sucesso a síntese de carbon dots, com luminescência no verde e tamanho médio inferior a $10 \mathrm{~nm}$, através do tratamento hidrotermal do extrato do bagaço de limão do tipo taiti, provenientes de diferentes cidades de Alagoas. As características estruturais e fotofísicas desses C-Dots foram similares, sugerindo assim, que são reprodutíveis. Outrossim, os carbon dots mostraram-se potenciais para aplicação em sensores de cromo (VI). Dessa forma, serão realizados estudos mais aprofundados para o desenvolvimento de uma sonda fluorescente para detecção desse metal em amostras sintéticas e reais. Adicionalmente, estudos de reprodutibilidade de C-Dots ainda são raros na literatura, e até essa data, não há relatos de trabalhos avaliando a reprodutibilidade de carbon dots derivados de limão.

\section{AGRADECIMENTOS}

Os autores agradecem à Universidade Federal de Alagoas (UFAL), à Coordenação de Aperfeiçoamento de Pessoal de Nível Superior (CAPES) pela concessão de bolsa de estudo para o desenvolvimento desse trabalho, ao Grupo de Catálise e Reatividade Química (GCaR) e ao Grupo de óptica e nanoscopia (GON) da UFAL pela disponibilização da infraestrutura para realização dos experimentos e caracterizações dos C-Dots.

\section{REFERÊNCIAS BIBLIOGRÁFICAS}

1. Li H, Kang Z, Liu Y, Lee S.-T. Carbon nanodots: synthesis, properties and applications. J Mater Chem. 2012;22(46):24230-24253. doi:10.1039/c2jm34690g

2. Xu X, Ray R, Gu Y, Ploehn HJ, Gearheart L, Raker K, Scrivens WA. Electrophoretic Analysis and Purification of Fluorescent Single-Walled Carbon Nanotube Fragments. J Am Chem Soc. 2004;126(40):12736-12737. doi:10.1021/ja040082h

3. Yuan F, Li S, Fan Z, Meng X, Fan L, Yang S. Shining carbon dots: Synthesis and biomedical and optoelectronic applications. Nano Today. 2016;11(5):565-586. doi:10.1016/j.nantod.2016.08.006

4. Liu ML, Chen BB, Li CM, Huang CZ. Carbon dots: synthesis, formation mechanism, fluorescence origin and sensing applications. Green Chem. 2019;21:449-471. doi:10.1039/c8gc02736f

5. Bu L, Peng J, Peng H, Liu S, Xiao H, Liu D, He Y. Fluorescent carbon dots for the sensitive detection of $\mathrm{Cr}(\mathrm{vi})$ in aqueous media and their application in test papers. RSC Advances. 2016;6(98):95469-95475. doi:10.1039/c6ra19977a

6. Sahu S, Behera B, Maiti TK, Mohapatra S. Simple one-step synthesis of highly luminescent carbon dots from orange juice: application as excellent bio-imaging agents. Chem Comm. 2012;48(70):8835-8837. doi:10.1039/c2cc33796g.

7. Ding H, Yu S-B, Wei J-S, Xiong H-M. Full-Color Light-Emitting Carbon Dots with a Surface-StateControlled Luminescence Mechanism. ACS Nano. 2015;10(1):484-491. doi:10.1021/acsnano.5b05406

8. Liu Z, Zou H, Wang N, Yang T, Peng Z, Wang J, Huang C. Photoluminescence of carbon quantum dots: coarsely adjusted by quantum confinement effects and finely by surface trap states. Sci China Chem. 2018;61(4):490-496. doi:10.1007/s11426-017-9172-0 
9. Essner JB, Kist JA, Polo-Parada L, Baker GA. Artifacts and Errors Associated with the Ubiquitous Presence of Fluorescent Impurities in Carbon Nanodots. Chem Mater. 2018;30(6):1878-1887. doi:10.1021/acs.chemmater.7b04446

10. Schneider J, Reckmeier CJ, Xiong Y, von Seckendorff M, Susha AS, Kasák P, Rogach AL. Molecular Fluorescence in Citric Acid-Based Carbon Dots. J Phys Chem C. 2017;121(3):2014-2022. doi:10.1021/acs.jpcc.6b12519

11. Sharma V, Tiwari P, Mobin SM. Sustainable carbon-dots: recent advances in green carbon dots for sensing and bioimaging. J Mater Chem B. 2017;5(45):8904-8924. doi:10.1039/c7tb02484c

12. Hsu P-C, Shih Z-Y, Lee C-H, Chang H-T. Synthesis and analytical applications of photoluminescent carbon nanodots. Green Chemistry. 2012;14(4):917-920. doi:10.1039/c2gc16451

13. Ding H, Zhou X, Qin B, Zhou Z, Zhao Y. Highly fluorescent near-infrared emitting carbon dots derived from lemon juice and its bioimaging application. J Lumin. 2019;211:298304. doi:10.1016/j.jlumin.2019.03.064

14. Ding H, Ji Y, Wei J-S, Gao Q-Y, Zhou Z-Y, Xiong H-M. Facile synthesis of red-emitting carbon dots from pulp-free lemon juice for bioimaging. J Mater Chem B. 2017;5(26):52725277. doi:10.1039/c7tb01130j

15. Basavaiah K, Tadesse A, RamaDevi D, Hagos M, Battu G. Facile Green Synthesis of Fluorescent Carbon Quantum Dots from Citrus Lemon Juice for Live Cell Imaging. Asian J Nanosci Mater. 2018;36-46. doi: 10.26655/ajnanomat.2018.1.5

16. Tyagi A, Tripathi KM, Singh N, Choudhary S, Gupta RK. Green synthesis of carbon quantum dots from lemon peel waste: applications in sensing and photocatalysis. RSC Advances. 2016;6(76):7242372432. doi:10.1039/c6ra10488f

17. He M, Zhang J, Wang H, Kong Y, Xiao Y, Xu W. Material and Optical Properties of Fluorescent Carbon Quantum Dots Fabricated from Lemon Juice via Hydrothermal Reaction. Nanoscale Res Lett. 2018;13(1). doi:10.1186/s11671-018-2581-7

18. Mendonça L, Conceição A da, Piedade J, Carvalho VD de, Theodoro VC de A. Caracterização da composição química e do rendimento dos resíduos industriais do limão Tahiti (Citrus latifolia Tanaka). Ciência Tecnol Aliment. 2006;26(4):870-874.doi: 10.1590/S0101-20612006000400025

19. Zhang J, Yuan Y, Liang G, Yu S-H. Scale-Up Synthesis of Fragrant Nitrogen-Doped Carbon Dots from Bee Pollens for Bioimaging and Catalysis. Adv Sci. 2015;2(4):15000021500008. doi:10.1002/advs.201500002

20. Meiling TT, Cywiński PJ, Bald I. White carbon: Fluorescent carbon nanoparticles with tunable quantum yield in a reproducible green synthesis. Sci Rep. 2016;6(1):28557-28565. doi:10.1038/srep28557

21. Fayad S, Ramos B, Minatti E, Soldi V. Nanopartículas obtidas a partir da proteína isolada de soja (spi) pelo método de coacervação em água: efeito da força iônica e das concentrações de proteína e surfactante. In: Anais do $10^{\circ}$ Congresso Brasileiro de Polímeros; Outubro de 2009; Foz do Iguaçu, PR.

22. Hoan BT, Tam PD, Pham V-H. Green Synthesis of Highly Luminescent Carbon Quantum Dots from Lemon Juice. J Nanotechnology. 2019;1-9. doi: 10.1155/2019/2852816 